\title{
Borel extensions of Baire measures
}

\author{
by
}

\author{
J. M. Aldaz (Madrid)
}

\begin{abstract}
We show that in a countably metacompact space, if a Baire measure admits a Borel extension, then it admits a regular Borel extension. We also prove that under the special axiom \& there is a Dowker space which is quasi-Mařík but not Mařík, answering a question of $\mathrm{H}$. Ohta and $\mathrm{K}$. Tamano, and under $P(c)$, that there is a Mařík Dowker space, answering a question of W. Adamski. We answer further questions of H. Ohta and K. Tamano by showing that the union of a Mařík space and a compact space is Mařík, that under "c is real-valued measurable", a Baire subset of a Mařík space need not be Mařík, and finally, that the preimage of a Mařík space under an open perfect map is Mařík.
\end{abstract}

1. Introduction. The Borel sets are the $\sigma$-algebra generated by the open sets of a topological space, and the Baire sets are the smallest $\sigma$ algebra making all real-valued continuous functions measurable. The Borel extension problem asks: Given a Baire measure, when can it be extended to a Borel measure? Whenever one deals with Baire measures on a topological space, it is assumed that the space is completely regular and Hausdorff, so there are enough continuous functions to separate points and closed sets. In 1957 (see [Ma]), J. Mařík proved that all normal, countably paracompact spaces have the following property: Every Baire measure extends to a regular Borel measure. Spaces which have this property have come to be known as Mařík spaces. We shall see later that if a normal space is not countably paracompact, then anything can happen. While answering questions from the survey paper [Wh1], H. Ohta and K. Tamano introduced in [OT] the notion of quasi-Mařík spaces, the spaces for which every Baire measure has a Borel extension, not necessarily regular. They noted that neglecting the regularity of the extension allowed them to get much stronger results, and wondered whether every quasi-Mařík space must be Mařík. We prove, in Section 2, that this is the case if the space is countably metacompact. This leads us, in order to search for an example of a quasi-Mařík space which

1991 Mathematics Subject Classification: 28C15, 54C50.

Key words and phrases: Mařík, quasi-Mařík, countably metacompact, Dowker. 
is not Maŕík, to the class of spaces that are not countably metacompact. When normal, such spaces are called Dowker, and almost Dowker if they are just regular.

Dowker spaces have been an object of continued interest among topologists. A standard hypothesis (which later on would turn out to be unnecessary) in certain homotopy extension theorems was that the product of the space $X$ with the closed unit interval be normal. H. Dowker showed (in 1951, see [Do]) that for a normal space $X$, this happens iff $X$ is countably paracompact (countable paracompactness is equivalent, in the presence of normality, to countable metacompactness). So the question arose as to whether there were normal not countably paracompact (i.e., Dowker) spaces. The first example in ZFC of a Dowker space was given by Mary Ellen Rudin in [Ru2] (she had previously shown, in 1955, that the existence of a Suslin line implied the existence of a Dowker space). Despite the fact that the original motivation for the study of Dowker spaces has disappeared, efforts to construct Dowker spaces with prescribed characteristics continue to this day.

With respect to the question of $\mathrm{H}$. Ohta and K. Tamano mentioned above, we shall show, using a Dowker space obtained from the special axiom \&, that there is a quasi-Mařík space which is not Mařík. Furthermore, W. Adamski has asked whether there is a Dowker space which is Mařík. We answer positively, under the axiom $P(c)$. We also note (Remarks 2.4) that assuming $\boldsymbol{h}$, a conjecture of A. W. Hager, G. D. Reynolds and M. D. Rice (see $[\mathrm{HRR}]$ ) is false (they suggest that if a space $X$ has no closed discrete subset of measurable power and satisfies $\mathrm{Bo}(X)=\mathrm{Ba}(X)$, then $X$ is realcompact). Likewise, a question of $\mathrm{R}$. B. Kirk (in [Ki], p. 338) receives a negative answer. He asks whether the condition "every Baire measure has a unique regular Borel extension" implies that the space is measure compact.

In Section 3 we further investigate the topological properties of Mařík and quasi-Mařík spaces, and answer the following questions of H. Ohta and K. Tamano: Must the union of a Mařík space and a compact space be Mařík? (the answer is yes); must a Baire subset of a Mařík space be Mařík? (under " $c$ is real valued measurable", no); must the preimage of a Marrík space under an open perfect map be Mařík? (yes; in particular, the product of a compact space with a Mařík space is Mařík).

2. Extension of measures. Let $X$ be a completely regular Hausdorff space (only such spaces will be considered here) and let $\mathcal{Z}(X)$ be the collection of zero sets of continuous real-valued functions defined on $X$. The smallest $\sigma$-algebra making all such functions measurable is called the Baire sets. It coincides with $\sigma(\mathcal{Z}(X))$, the $\sigma$-algebra generated by $\mathcal{Z}(X)$. By a measure, we mean a countably additive finite measure. A measure defined 
on $\sigma(\mathcal{Z}(X))$ is called a Baire measure. If a topological fact is mentioned without a specific reference, then it can be found in [Eng]. A topological space $X$ is countably metacompact if every countable open cover of $X$ has a point-finite open refinement, and countably paracompact if every countable open cover of $X$ has a locally finite open refinement. By [Ish], $X$ is countably metacompact iff for every sequence $\left\{C_{n}\right\}$ of closed sets satisfying $C_{n} \downarrow \emptyset$ (i.e., $C_{n+1} \subset C_{n}$ and $\bigcap_{n} C_{n}=\emptyset$ ), there is a sequence $\left\{O_{n}\right\}$ of open sets such that for every $n, C_{n} \subset O_{n}$, and $O_{n} \downarrow \emptyset$, while $X$ is countably paracompact iff for every sequence $\left\{C_{n}\right\}$ of closed sets satisfying $C_{n} \downarrow \emptyset$, there is a sequence $\left\{O_{n}\right\}$ of open sets such that for every $n, C_{n} \subset O_{n}$, and $\bar{O}_{n} \downarrow \emptyset$. When $X$ is normal, countable metacompactness is equivalent to countable paracompactness. A topological space is Mařik if every Baire measure has a regular Borel extension, and quasi-Mařik if every Baire measure has a Borel extension.

We begin with a result which shows why the notion of countable metacompactness is relevant to the topic of Mařík and quasi-Mařík spaces.

\subsection{TheOREM. A countably metacompact quasi-Mařík space is Mařik.}

Proof. Let $X$ be a countably metacompact quasi-Mařík space, and let $\mu$ be a Baire measure on $X$. Let $\tau$ be any Borel extension of $\mu$, and let $\mathcal{A}$ be the algebra on $X$ generated by the open sets. By Corollary 2.12 of [Lem] there exists a finitely additive regular measure $\nu$ on $\mathcal{A}$ with $\nu(X)=$ $\tau(X)$ and $\nu \geq \tau$ on the closed subsets of $X$ (the existence of such a $\nu$ follows by a typical Zorn Lemma argument. More general results in this line are Proposition 3.4 of [Pa] and Theorem 2.2 of [Ad1].) Furthermore, for every sequence $\left\{C_{n}\right\}$ of closed sets with $C_{n} \downarrow \emptyset$ there exists, by the countable metacompactness of $X$, a sequence $\left\{O_{n}\right\}$ of open sets with $C_{n} \subset$ $O_{n}$, and $O_{n} \downarrow \emptyset$. Thus $\lim _{n} \nu\left(C_{n}\right) \leq \lim _{n} \nu\left(O_{n}\right) \leq \lim _{n} \tau\left(O_{n}\right)=0$ since $\tau$ is countably additive. By the regularity of $\nu$, the same conclusion holds for arbitrary decreasing sequences $\left\{A_{n}\right\}$ of sets in $\mathcal{A}$ with $A_{n} \downarrow \emptyset$ : to see this, fix $\varepsilon>0$, and select for each $n$ a closed $C_{n} \subset A_{n}$ such that $\nu\left(A_{n} \backslash C_{n}\right)<2^{-n} \varepsilon$; since $\bigcap_{i=1}^{n} C_{i} \downarrow \emptyset$, we have $\lim _{n} \nu\left(\bigcap_{i=1}^{n} C_{i}\right)=0$ and thus $\lim _{n} \nu\left(A_{n}\right) \leq \varepsilon$. It follows from Carathéodory's Extension Theorem (or from Theorem 1 of $[\mathrm{T}])$ that $\nu$ extends to a regular Borel (countably additive) measure, which we also denote by $\nu$.

To see that $\nu$ extends $\mu$, it is enough to show (by the regularity of Baire measures) that for every zero set $Z, \mu(Z)=\nu(Z)$. So fix a zero set $Z_{1}$. Since $\nu$ dominates a Borel extension of $\mu$ on the closed sets, we have $\mu\left(Z_{1}\right) \leq \nu\left(Z_{1}\right)$. Suppose that $\mu\left(Z_{1}\right)<\nu\left(Z_{1}\right)$. Choose $\varepsilon>0$ with $\nu\left(Z_{1}\right)-\mu\left(Z_{1}\right)>\varepsilon$. Then we can find a zero set $Z_{2} \subset X \backslash Z_{1}$ such that $\mu\left(Z_{1}\right)+\mu\left(Z_{2}\right)>\mu(X)-\varepsilon$. But now $\nu\left(Z_{1}\right)+\nu\left(Z_{2}\right)>\mu\left(Z_{1}\right)+\varepsilon+\mu\left(Z_{2}\right)>\mu(X)=\nu(X)$, contradicting the additivity of $\nu$. 
A set $Y \subset X$ is said to be Baire embedded in $X$ if for every $A \in \operatorname{Ba}(Y)$ there exists a $B \in \mathrm{Ba}(X)$ such that $A=B \cap Y$. Theorem 2.1 allows one to strengthen previously known results if the space $X$ is countably metacompact. For instance, in $[\mathrm{OT}] \mathrm{H}$. Ohta and K. Tamano give the following definition: A set $S \subset X$ is a generalized Baire subset of $X$ if for every open set $O$ containing $S$, there exists a set $B \in \mathrm{Ba}(X)$ with $S \subset B \subset O$. Using this notion, they show that if $X=\bigcup_{n} X_{n}$, where each $X_{n}$ is a Mařík, Baire embedded, generalized Baire subset of $X$, then $X$ is a Mařík space (Theorem 4.9 of $[\mathrm{OT}]$ ). They also point out (Remark 4.12(2)) that if the assumption that each $X_{n}$ is a generalized Baire subset of $X$ is removed, then their proof shows that $X$ is still quasi-Mař́k. Now it is easy to check that to obtain this conclusion, it is enough that each $X_{n}$ be quasi-Mařík rather than Mařík. So from their proof and Theorem 2.1 one gets the following

2.2. Theorem. Let $X=\bigcup_{n} X_{n}$, where each $X_{n}$ is quasi-Mařik and Baire embedded in $X$. If $X$ is countably metacompact, then it is Mařik.

Of course, the usefulness of Theorem 2.1 (and the type of argument outlined above) will depend on how common or rare countably metacompact spaces turn out to be. A topological space is Dowker if it is normal but not countably paracompact, and almost Dowker if it is regular but not countably metacompact. For a long time there has been, up to small modifications, only one known example in ZFC of a Dowker space, namely M. E. Rudin's (see [Ru2]); recently two new Dowker spaces have been announced: see [Sz]. Almost Dowker spaces were introduced by B. M. Scott in [S]. They appear to be far more abundant than Dowker spaces, particularly among large products, as is pointed out at the end of our paper.

Next we give an example of a quasi-Mařík normal space that is not Mařík, under the special set-theoretic hypothesis $\boldsymbol{\alpha}$. In the version from [Ru1], states that for every limit ordinal $\alpha<\omega_{1}$ there is a sequence $S_{\alpha}$ (order isomorphic with $\omega$ ), cofinal in $\alpha$, such that every uncountable subset of $\omega_{1}$ contains some $S_{\alpha}$. We shall use the Dowker space appearing in [Ru1], 3.1(i), pp. 768-769, which is based on constructions due to A. J. Ostaszewski and P. de Caux. We briefly describe this space (for more details, see [Ru1]). Assume \&, and partition each $S_{\alpha}$ into infinite (disjoint) subsets $\left\{S_{\alpha i j n}\right.$ : $i, j, n \in \omega\}$. Define $U$ to be an open subset of the space $X=\omega_{1} \times \omega$ iff for every $(\alpha+j, n) \in U$ (where $\alpha$ is a limit ordinal and $j, n \in \omega$ ) and every $i \leq n$, there is a cofinite subset $S^{i}$ of $S_{\alpha i j n}$ such that $\left\{(\beta, i): i \in S^{i}\right\} \subset U$. This space is hereditarily normal and zero-dimensional. Furthermore, if we set $F_{n}:=\bigcup_{j \geq n} \omega_{1} \times\{j\}$, then each $F_{n}$ is closed, and given any open set $U$ with $F_{n} \subset U, U$ is cocountable (so $X$ is not countably metacompact). To prove that $U$ is cocountable one uses the fact that the closed subsets of each $\omega_{1} \times\{n\}$ with the subspace topology are either countable or cocountable. 
Since this will be useful later (for it implies that the Baire and the Borel subsets of $\omega_{1} \times\{n\}$ are the same), we go into more detail here. Suppose that $C$ is closed and uncountable in $\omega_{1} \times\{n\}$. By some $S_{\alpha}$ satisfies $S_{\alpha} \times\{n\} \subset C$. Then the closure of $S_{\alpha} \times\{n\}$ in $\omega_{1} \times\{n\}$ contains the set $\{(\beta, n): \beta \geq \alpha\}$ (just note that for all $j \in \omega$, we have $(\alpha+j, n) \in \overline{S_{\alpha} \times\{n\}}$, hence $(\alpha+\omega, n) \in$ $\overline{S_{\alpha} \times\{n\}}$, and repeat the argument), so $C$ is cocountable.

2.3. Theorem. Assume . Then there exists a normal quasi-Mařik space which is not Mař́k.

Proof. Consider the space $X=\omega_{1} \times \omega$ with the Dowker topology described above. For each $n \in \omega, \operatorname{Ba}\left(\omega_{1} \times\{n\}\right)$ is the $\sigma$-algebra of countable and cocountable sets, and furthermore, $\mathrm{Ba}\left(\omega_{1} \times\{n\}\right)=\mathrm{Bo}\left(\omega_{1} \times\{n\}\right)$. To see this, recall that every open set in $\omega_{1} \times\{n\}$ is either countable or cocountable. Since the collection of countable and cocountable sets of $\omega_{1} \times\{n\}$ is a $\sigma$-algebra which contains the open sets, it contains all the Borel sets. On the other hand, initial segments of $\omega_{1} \times\{n\}$ are open, and since they are countable, they can be expressed as a countable union of cozero sets, so they are cozero. It follows that every singleton is Baire, since it is the difference of two cozero sets. Hence, every countable subset of $\omega_{1} \times\{n\}$ is Baire.

The same reasoning shows that if $D$ is a countable subset of $\omega_{1}$, then $D \times \omega$ is Baire in $X$. Therefore each set $\omega_{1} \times\{n\}$ is Baire embedded in $X$ : For any countable subset $A=\left\{\left(\alpha_{k}, n\right): k \in \omega\right\} \subset \omega_{1} \times\{n\}$, we have $A=\left(\left\{\alpha_{k}: k \in \omega\right\} \times \omega\right) \cap \omega_{1} \times\{n\}$. Now $\left\{\alpha_{k}: k \in \omega\right\} \times \omega$ is a Baire subset of $\omega_{1} \times \omega$, so the claim follows.

By Theorem 4.9 and Remark 4.12(2) of [OT], if $X=\bigcup_{n} X_{n}$, where each $X_{n}$ is Mařík and Baire embedded in $X$, then $X$ is quasi-Mařík. Letting $X_{n}$ be $\omega_{1} \times\{n\}$, we find that $X=\bigcup_{n} \omega_{1} \times\{n\}$ is quasi-Mař́k, for each $\omega_{1} \times\{n\}$ is Baire embedded in $X$ and trivially Maŕík (as $\mathrm{Ba}\left(\omega_{1} \times\{n\}\right)=\operatorname{Bo}\left(\omega_{1} \times\{n\}\right)$ ).

Finally, in order to prove that $X$ is not Mařík, we define a Baire measure $\nu$ on $X$ by declaring $\nu(B)=1$ if $B \cap \omega_{1} \times\{0\}$ is cocountable, and $\nu(B)=0$ if $B \cap \omega_{1} \times\{0\}$ is countable. Let $\mu$ be any Borel extension of $\nu$, and set $F_{n}:=\bigcup_{j \geq n} \omega_{1} \times\{j\}$. Then $\lim \mu\left(F_{n}\right)=0$, but any open set $O$ containing any $F_{n}$ is cocountable, so $\mu(O)=1$ and therefore $\mu$ is not regular.

2.4. R e marks. In [HRR], p. 140, it is conjectured that if a space $X$ has no closed discrete subset of measurable power and satisfies $\mathrm{Bo}(X)=\mathrm{Ba}(X)$, then $X$ is realcompact. However, the subset $\omega_{1} \times\{0\}$ of the Dowker space $X$ considered above (or the Ostaszewski line, see [Ost]) has nonmeasurable cardinality and satisfies $\operatorname{Bo}\left(\omega_{1} \times\{0\}\right)=\mathrm{Ba}\left(\omega_{1} \times\{0\}\right)$, so under of the conjecture is false. This remark appears not to have been made before.

R. B. Kirk has asked ([Ki], p. 338) whether the condition that every Baire measure on a space $X$ have a unique regular Borel extension entails 
that $X$ is measure compact. The same example as before $\left(X=\omega_{1} \times\{0\}\right)$ shows that the answer is no: Since $\operatorname{Bo}(X)=\mathrm{Ba}(X)$, trivially every Baire measure has a unique regular Borel extension, but the space is not even realcompact.

The fact that there are Dowker non-Mařík spaces is well known, and we have shown that a Dowker space can be quasi-Mařík while failing to be Mař́k. Going further in this direction (see [Ad2], Remark 2.4.c, p. 89) W. Adamski has asked whether a Dowker space can be Mař́k. Under the special set-theoretic assumption $P(c)$ there exists a Dowker space where every Baire measure is discrete (Theorem 2.5 below), so it is trivially Mařík. This also shows that countable metacompactness is not a necessary condition for a quasi-Mařík space to be Mařík. The letter $c$ stands for the cardinality of the continuum, and $P(c)$ denotes the following statement: Let $\mathcal{A}$ be any collection of fewer than $c$ subsets of the natural numbers, such that whenever $F \subset \mathcal{A}$ is finite, $\bigcap F$ is infinite. Then there exists an infinite set $B \subset \mathbb{N}$ with $B \backslash A$ finite for every $A \in \mathcal{A}$. A generalized Luzin subset of the Cantor space $2^{\omega}$ is a subset of $2^{\omega}$ with cardinality $c$ and such that its intersection with any set of the first category has cardinality less than $c$. The existence of a generalized Luzin set follows from $P(c)$ (see [Be], pp. 152-153). Recall that a Borel measure is continuous if every point has measure zero, and discrete if there is a countable set with full measure. Every Borel measure can be decomposed into a continuous and a discrete part.

2.5. Theorem. Assume $P(c)$. Then there exists a Dowker space which admits only discrete Baire measures.

Proof. By [Be], $P(c)$ entails that there is a Dowker space $(X, \tau)$ such that $X$ is contained in a generalized Luzin subset $L$ of $2^{\omega}$, and the topology $\tau$ refines the euclidean (or product) topology $e$. Now on $(X, e)$ the Baire and the Borel sets coincide. Therefore any Baire measure $\mu$ on $(X, \tau)$ defines by restriction a Baire measure (and hence a Borel measure) on $(X, e)$. We extend this measure to $2^{\omega}$ by setting $\nu(B):=\mu(B \cap X)$ for every Borel set $B \subset 2^{\omega}$. Write $\nu=\nu_{\mathrm{c}}+\nu_{\mathrm{d}}$, where $\nu_{\mathrm{c}}$ and $\nu_{\mathrm{d}}$ are respectively the continuous and discrete parts of $\nu$. There exists an $F_{\sigma}$ subset $F$ of $2^{\omega}$ such that $F$ is of the first category and $\nu(F)=\nu\left(2^{\omega}\right)$. Martin's Axiom for $\sigma$-centered posets (MA $(\sigma$-centered), see $[\mathrm{F} 1]$ or $[\mathrm{W}]$ for the definition) implies that the union of less than $c$ closed sets of measure zero has outer measure zero, for every continuous Borel measure on $2^{\omega}$ (Corollary $22-\mathrm{H}$ of [F1]). Since $P(c)$ is equivalent to $\operatorname{MA}(\sigma$-centered) (by $[\mathrm{Be}])$, and $L$ is generalized Luzin, it follows that the cardinality of $L \cap F$ is less than $c$ and thus the $\nu_{\mathrm{c}}$-outer measure of $L \cap F$ is zero. But $\nu$ assigns full outer measure to $L$, so $\nu_{\mathrm{c}}$ is identically zero and $\nu$ purely discrete. Therefore so is $\mu$. 
2.6. R e mark. A Baire measure $\mu$ is $\tau$-smooth (or $\tau$-additive) if for every collection of cozero sets $\mathcal{C}=\left\{O_{\alpha}\right\}$, closed under finite unions and such that $\bigcup \mathcal{C}=X$, we have $\mu(X)=\sup _{\alpha} \mu\left(O_{\alpha}\right)$. If we replace $X$ by an arbitrary cozero set, the definition so obtained is equivalent to the previous one. A space $X$ is measure compact if every Baire measure on $X$ is $\tau$-smooth, and strongly measure compact if every Baire measure $\nu$ on $X$ is tight, i.e., for every $\varepsilon>0$ there exists a compact set $K$ with $\bar{\nu}(K)>\nu(X)-\varepsilon$. Problem 9.10 of [Wh1] asks whether there is a measure compact space where the closed sets are not sequentially dominated by the cozero sets (this condition is stronger than countable metacompactness). H. Ohta and K. Tamano have answered this affirmatively in $[\mathrm{OT}]$ by giving three different examples. These spaces are nonnormal; one of them is locally compact and does not require any set-theoretic assumptions beyond ZFC. With stronger assumptions (ZFC + $P(c)$ ), Bell's Dowker space provides an example which is strongly measure compact and normal.

3. Topological properties of Mařík and quasi-Mařík spaces. It is often of interest to know how properties of a space behave under different topological operations. Regarding unions, for instance, it is known that the union of a measure compact space with a compact space is measure compact, while the union of a realcompact space and a compact space is realcompact. In this context, H. Ohta and K. Tamano show that the union of two Mařík spaces need not be quasi-Mařík, even if one is a cozero set and the other a zero set ([OT], Remark 4.12(1)). They also ask (Question 4.13) whether the union of a Mařík space with a compact space is Mařík. We answer this affirmatively (Corollary 3.4), and show that the corresponding statement is again true for quasi-Mařík spaces. The next lemma is obvious.

3.1. Lemma. A Baire embedded, Baire subset of a quasi-Mařik space is quasi-Mařik.

This lemma fails if the condition that the subspace be a Baire subset is omitted. To see that a Baire embedded subset of a Mař́k space need not be quasi-Mařík, consider a non-quasi-Mařík set in its Stone-Čech compactification.

Recall that a Borel measure $\nu$ is $\tau$-smooth if for every collection of open sets $\mathcal{C}=\left\{O_{\alpha}\right\}$, closed under finite unions, $\mu(\bigcup \mathcal{C})=\sup _{\alpha} \mu\left(O_{\alpha}\right)$. It is well known that every $\tau$-smooth Baire measure $\mu$ (see Remark 2.6 for the definition) has a unique $\tau$-smooth Borel extension $\nu$. Furthermore, every $\tau$-smooth Borel measure on a regular space is regular.

3.2. Theorem. Let $X=Y \cup L$, where $Y$ is Mařik (respectively quasiMařk ) and $L$ is Lindelöf. If for every zero subset $Z$ of $X$ disjoint from $L$ 
there exists another zero set $S$ with $L \subset S$ and $S \cap Z=\emptyset$, then $X$ is Mařik (respectively quasi-Mařik).

Pr o of. Let $Y$ be Mařík and let $X=Y \cup L$, where $L$ is a Lindelöf set such that for every zero subset $Z$ with $L \cap Z=\emptyset$, there exists another zero set $S$ with $L \subset S$ and $S \cap Z=\emptyset$. Given a Baire measure $\mu$ on $X$, we can decompose $\mu$ into a purely countably additive part and a $\tau$-smooth part (Theorem 4.3 of $[\mathrm{Kn}])$. Since the $\tau$-smooth part always extends to a regular ( $\tau$-smooth) Borel measure, we may assume that $\mu$ is purely countably additive, i.e., the only $\tau$-smooth minorant of $\mu$ is the zero measure. Define a Baire measure $\nu$ on $X$ by setting $\nu(B)=\mu^{*}(L \cap B)$ for $B \in \mathrm{Ba}(X)$. Given any collection $\left\{O_{\alpha}\right\}$ of cozero sets with $\bigcup_{\alpha} O_{\alpha}=X$, there is a countable subcollection $\left\{O_{n}: n \in \mathbb{N}\right\}$ which covers $L$, whence $\nu(X)=\nu\left(\bigcup_{n} O_{n}\right)$. Therefore $\nu$ is $\tau$-smooth, and since it is also a minorant of $\mu$, it must be identically zero. It follows that the $\mu$-outer measure of $L$ is zero. Now for each $n \in \mathbb{N}$ select a cozero set $O_{n}$ such that $L \subset O_{n}$ and $\mu\left(O_{n}\right)<1 / n$. By assumption, given $n \in \mathbb{N}$ we can choose a cozero set $U_{n}$ with $O_{n}^{\mathrm{c}} \subset U_{n}$ and $L \cap U_{n}=\emptyset$. Then $O:=\bigcup_{n} U_{n}$ is a cozero subset of $X$ with $\mu(O)=\mu(X)$ and $O \cap L=\emptyset$. Since $O$ is Baire embedded in $X$, it follows that $\mu_{O}(O \cap B):=\mu(B)$ defines a Baire measure on $O$. But $O$ is a cozero subset of the Mařík space $Y$, so it is Mařík (Corollary 4.3 of $[\mathrm{OT}]$ ). Let $\nu_{O}$ be a regular Borel extension of $\mu_{O}$. Setting $\nu(B):=\nu_{O}(O \cap B)$ for all $B \in \operatorname{Bo}(X)$, we obtain a Borel extension of $\mu$. To see that $\nu$ is regular, fix $\varepsilon>0$ and select a Borel set $B \subset X$. Then $B=(B \cap O) \cup\left(B \cap O^{\mathrm{c}}\right)$. Let $V \subset O$ be an open set such that $B \cap O \subset V$ and $\nu_{O}(V)-\varepsilon / 2<\nu_{O}(B \cap O)$. Select a cozero subset $W$ of $X$ with $O^{\text {c }} \subset W$ and $\mu(W)<\varepsilon / 2$. Then we have $B \subset V \cup W$ and $\nu(V \cup W)-\varepsilon \leq \nu(V)+\nu(W)-\varepsilon=\nu_{O}(V)+\mu(W)-\varepsilon<\nu(B)$, so $\nu$ is regular.

The quasi-Mařík case is proven in the same fashion, save that we use our Lemma 3.1 instead of Corollary 4.3 from [OT].

3.3. R e mark. If $Y \subset X$ and $\nu$ is a regular Borel measure on $Y$, it does not automatically follow that the extension to a Borel measure on $X$ given by $\nu_{X}(B):=\nu(Y \cap B)$ for all $B \in \mathrm{Bo}(X)$ is regular. Consider, for instance, the Dieudonné measure on $\omega_{1}$ and its extension to $\omega_{1}+1$.

3.4. Corollary. The union of a Mařik space with a compact space is Mařik. Likewise, the union of a quasi-Mařik space with a compact space is quasi-Mařik.

Proof. Let $K$ be compact, and let $X=Y \cup K$, where $Y$ is either Mařík or quasi-Mař́k. Let $Z$ be a zero subset of $X$ with $K \cap Z=\emptyset$. For each $x \in K$ select a continuous function $f_{x}: X \rightarrow[0,1]$ satisfying $f_{x}^{-1}(\{0\})=Z$ and $f_{x}(x)=1$. Then the open cover $\left\{f_{x}^{-1}((1 / 2,1]): x \in K\right\}$ of $K$ has a finite sub- 
cover $\left\{f_{i}^{-1}((1 / 2,1]): i=1, \ldots, n\right\}$. But now the zero set $\bigcup_{i=1}^{n} f_{i}^{-1}([1 / 2,1])$ contains $K$ and is disjoint from $Z$, so the conclusions follow from the previous theorem.

3.5. Remarks. The proof of Theorem 3.2 can be easily modified to cover the case where $L$, instead of Lindelöf, is measure compact and Baire embedded in $X$. If $X$ is already known to be quasi-Mařík, then it is enough that $L$ be quasi-Mařík and $Y$ be $F_{\sigma}$ in $X$ to conclude that $X$ is Mař́k (Theorem 3.6 below).

If $L$ is a Lindelöf closed subset of $X$, and $Y$ is discrete (thus trivially Mařík), then a stronger conclusion holds: $X$ is not just Mařík but paracompact (see [Ka], p. 1251). On the other hand, if $L$ is simply Lindelöf, with no additional conditions, the result fails: Mrówka's space $\Psi$ (see [GJ], 5I, p. 79) is the union of two discrete spaces, one of cardinality less than or equal to $c$, and the other countable (hence Lindelöf and $\sigma$-compact). As noted in [Ad3] and $[\mathrm{OT}]$, under the assumption that $c$ is not real-valued measurable $\Psi$ is not quasi-Mařík, for all its subsets are Borel, but the space is not realcompact.

3.6. Theorem. Let $Y$ be Mařik and let $M$ be measure compact. If $X=$ $Y \cup M$ is quasi-Mařik and $Y$ is $F_{\sigma}$ in $X$, then $X$ is Mařik.

Proof. Let $\mu$ be a Baire measure on $X$. As in the proof of Theorem 3.2, we may assume that $\mu$ is purely countably additive. Let $\nu$ be a Borel extension of $\mu$. We obtain a Borel measure $\nu_{M}$ on $M$ by setting $\nu_{M}(B)=$ $\nu^{*}(M \cap B)$ for $B \in \mathrm{Bo}(M)$. The restriction of $\nu_{M}$ to $\mathrm{Ba}(M)$ is $\tau$-smooth, and hence so is the Baire measure $\widehat{\nu}$ on $X$ given by $\widehat{\nu}(B)=\nu_{M}(B \cap M)$. But $\widehat{\nu}$ is a minorant of $\mu$, so it is identically zero. Thus there is a Borel subset $E$ of $X$ with $\nu(E)=\nu(X)$ and $E \subset Y \backslash M$. Define now a Baire measure $\gamma$ on $Y$ as follows: For $B \in \mathrm{Ba}(Y), \gamma(B)=\nu(E \cap B)$. Since $Y$ is Mař́k, $\gamma$ has a regular Borel extension which we also denote by $\gamma$. The Borel measure on $X$ defined by $\gamma_{X}(B)=\gamma(Y \cap B)$, for every Borel set $B \subset X$, is an extension of $\mu$, since if $H \in \mathrm{Ba}(X)$, then $\mu(H)=\nu(H)=\nu(E \cap H)=\gamma(Y \cap H)=\gamma_{X}(H)$. Finally, it is easy to see that $\gamma_{X}$ must be regular, for $Y$ is $F_{\sigma}$ in $X$ and $\gamma$ is regular on $Y$.

As noted before, H. Ohta and K. Tamano have shown (in [OT], Theorem 4.1) that a generalized Baire subset of a Mařík space is Mař́k provided that it is Baire embedded. They also ask whether this condition can be removed (Remark 4.4-2, p. 409 of [OT]). If we assume that $c$ is real-valued measurable, then there exists a Baire (and not just generalized Baire) subset of a Mařík space which is not quasi-Mařík (Theorem 3.8 below).

The symbol $\mathbb{R}^{2}$ will be used to denote the plane with the euclidean topology, and $\mathbb{R}_{\ell}^{2}$ for the plane with the Sorgenfrey topology (i.e., the topology generated by the semiopen rectangles $[a, b) \times[c, d)$ ). Under the assumption 
that $c$ is not real-valued measurable, the Sorgenfrey plane is a typical example of a non-quasi-Mařík space. However, if the opposite assumption is made, then it is Mařík, as will be shown next.

3.7. Theorem. Assume that $c$ is real-valued measurable. Then $\mathbb{R}_{\ell}^{2}$ is Mařik.

Pr o of. Let $\mu$ be a Baire measure on $\mathbb{R}_{\ell}^{2}$. We may, without loss of generality, assume that $\mu$ is continuous and $\mu\left(\mathbb{R}_{\ell}^{2}\right)=1$. Then $\mu$ is a continuous Borel probability measure on $\mathbb{R}^{2}$, since $\mathrm{Bo}\left(\mathbb{R}^{2}\right)=\mathrm{Ba}\left(\mathbb{R}_{\ell}^{2}\right)$, by Theorem 2.1 of $[\mathrm{Ba}]$. Hence there exists a measure preserving homeomorphism $h: E \rightarrow \mathbb{P}$, where $E$ is a $G_{\delta}$ subset of the euclidean plane with $\mu(E)=1$, and $\mathbb{P}$ is the irrational numbers in $[0,1]$ with Lebesgue measure $\lambda$ (see $[\mathrm{Ox}]$, Theorem 2). The assumption that $c$ is real-valued measurable is equivalent to the hypothesis that Lebesgue measure can be extended to all subsets of $\mathbb{P}$ (by [U], or Theorem $1 \mathrm{D}(\mathrm{e})$ of [F2]). Let $\nu$ denote such an extension. Then $\nu h^{-1}$ induces a Borel measure on $\mathbb{R}_{\ell}^{2}$ which extends $\mu$, so $\mathbb{R}_{\ell}^{2}$ is quasi-Mařík. But $\mathbb{R}_{\ell}^{2}$ is countably metacompact (see [SS], Example 84 and p. 190), whence it is Mařík, by Theorem 2.1.

In order to answer the question of $\mathrm{H}$. Ohta and $\mathrm{K}$. Tamano regarding whether or not the condition "Baire embedded" can be omitted from their Theorem 4.1, the idea is to use the previous result as follows: Assume that $c$ is real-valued measurable. For convenience, we shall consider the unit square $[0,1]^{2}$ with the Sorgenfrey topology, rather than the Sorgenfrey plane. Let $\lambda$ be Lebesgue linear measure on the negative diagonal $D$, and let $E$ be nonmeasurable of least possible cardinality. Then $\nu(B):=\lambda^{*}(E \cap B)$ is a Baire measure on the square. Let $\mu$ be any Borel extension of $\nu$. Theorem 14.7(ii) of $[\mathrm{Ku}]$ entails that $\mu(E)=0$. If we modify the topology on $D$ so that it is coarse enough for $\nu$ to be a Baire measure on $D$, but rich enough so that all sets in $D$ are Borel and $E$ is Baire, then $\nu$ will have no Borel extension. To achieve this result we add new points and change the basic neighborhoods of points in $E$. There are several ways of doing so, for example, by using a Cantor tree type of construction, or by considering two Sorgenfrey squares, perpendicular along their negative diagonals. We have chosen a third way, refining the topology of the unit cube $[0,1]^{3}$ in euclidean space. We use the vector notation $\mathbf{x}$ to denote points from $[0,1]^{3}$.

3.8. Theorem. Assume that $c$ is real-valued measurable. Then there exists a Baire subset of a Mařik space which is not quasi-Mařik.

Proof. Let $\left([0,1]^{3}, \mathcal{T}_{1}\right)$ be the unit cube with the euclidean topology. On the set $[0,1]^{3}$ we define a second, finer topology $\mathcal{T}_{2}$ by declaring the "upper part" $U=[0,1] \times[0,1] \times(0,1]$ and the "bottom" $B=[0,1] \times$ $[0,1] \times\{0\}$ clopen, and giving to the points in $U$ the euclidean topol- 
ogy, while the points in $B$ have the Sorgenfrey plane topology (generated by the sets $[a, b) \times[c, d) \times\{0\})$. Note that since the Baire sets of the Sorgenfrey plane coincide with the Borel sets of the euclidean plane, the Baire sets of $\left([0,1]^{3}, \mathcal{T}_{2}\right)$ and the Borel sets of $\left([0,1]^{3}, \mathcal{T}_{1}\right)$ are also identical. But $\mathrm{Ba}\left([0,1]^{3}, \mathcal{T}_{1}\right)=\operatorname{Bo}\left([0,1]^{3}, \mathcal{T}_{1}\right)$, so given any (completely regular) intermediate topology $\mathcal{T}_{3}$, finer than the euclidean topology but coarser than $\mathcal{T}_{2}$, we have $\mathrm{Ba}\left([0,1]^{3}, \mathcal{T}_{3}\right)=\operatorname{Bo}\left([0,1]^{3}, \mathcal{T}_{1}\right)$. Now $\left([0,1]^{3}, \mathcal{T}_{2}\right)$ is Mař́k, for it is the topological sum of two Mař́k spaces, namely $T$ (which has the euclidean topology) and $B$ (by Theorem 3.7, using the assumption that $c$ is real-valued measurable; in this regard there is no difference between the Sorgenfrey plane and the Sorgenfrey unit square). It follows that $\left([0,1]^{3}, \mathcal{T}_{3}\right)$ is also Mař́k. Denote by $D$ the "negative diagonal" of $B$, i.e., $D:=\{(x, 1-x, 0): x \in[0,1]\}$. Next we construct a completely regular topology $\mathcal{T}_{3}$ on $[0,1]^{3}$ with $\mathcal{T}_{1} \subset \mathcal{T}_{3} \subset \mathcal{T}_{2}$, such that the Baire subset $T \cup D$ fails to be quasi-Mařík.

Let $E \subset D$ be a nonmeasurable subset (with respect to linear Lebesgue measure) of least possible cardinality. To define $\mathcal{T}_{3}$, we give to the points in $T$ the euclidean topology, and to the points in $B \backslash E$ the Sorgenfrey plane topology (so the basic neighborhoods of the points in the complement of $E$ are the same as in $\mathcal{T}_{2}$ ). Finally, we assign to the points in $E$ a threedimensional variation of Nemytski1's tangent disc topology (see Example 82 of $[\mathrm{SS}])$. Denote by $V(\mathbf{x}, n)$ the euclidean open ball contained in $T$, of radius $1 / n$ and tangent to $B$ at the point $\mathbf{x}$, and by $S(\mathbf{x}, n)$ the set $[x, x+$ $1 / n) \times[y, y+1 / n) \times\{0\}$, where $\mathbf{x}=(x, y, 0)$. For each $\mathbf{x} \in E$, the basic neighborhoods of $\mathbf{x}$ are the sets $V(\mathbf{x}, n) \cup S(\mathbf{x}, n)$. Clearly, $\mathcal{T}_{1} \subset \mathcal{T}_{3} \subset \mathcal{T}_{2}$. To check that $\left([0,1]^{3}, \mathcal{T}_{3}\right)$ is completely regular, suppose that $C$ is closed and $\mathbf{x} \notin C$. If $\mathbf{x} \in T$, then there is a euclidean open ball centered at $\mathbf{x}$ which misses $C$, so any continuous real-valued function which is zero at $\mathbf{x}$ and one off the ball will separate $\mathbf{x}$ and $C$. Likewise, if $\mathbf{x} \in B \backslash E$, there exists an $n \in \mathbb{N}$ such that $S(\mathbf{x}, n) \cap C=\emptyset$. Then the function which takes the value zero on $S(\mathbf{x}, n)$ and one elsewhere is continuous and separates $\mathbf{x}$ and $C$. Finally, if $\mathbf{x}=\left(x_{0}, 1-x_{0}, 0\right) \in E$, there exists an $n \in \mathbb{N}$ such that $(V(\mathbf{x}, n) \cup S(\mathbf{x}, n)) \cap C=\emptyset$. Let $f=1$ off $V(\mathbf{x}, n) \cup S(\mathbf{x}, n)$, zero on $S(\mathbf{x}, n)$ and $f(x, y, z)=n\left[\left(x-x_{0}\right)^{2}+\left(y+x_{0}-1\right)^{2}+z^{2}\right] /(2 z)$ for $(x, y, z) \in$ $V(\mathbf{x}, n)$. Then $f$ is continuous and separates $\mathbf{x}$ and $C$. Therefore $\left([0,1]^{3}, \mathcal{T}_{3}\right)$ is completely regular.

Note that $T \cup D$ is the union of a cozero set and a zero set in $\left([0,1]^{3}, \mathcal{T}_{1}\right)$, hence also Baire in $\left([0,1]^{3}, \mathcal{T}_{3}\right)$. Next we determine what are the Baire sets of $T \cup D$ with the subspace topology from $\left([0,1]^{3}, \mathcal{T}_{3}\right)$. The Baire subsets of $T$ are the euclidean Borel sets, and $D \backslash E$ has the discrete topology, so all its subsets are Baire. As for $E$, it is a zero subset of $T \cup D$. Furthermore, if $Z$ is a zero subset of $E$, then $Z$ is a Borel subset of $E$ with the euclidean 
topology. The proof of this fact is essentially the same as the one given for the Sorgenfrey plane in Lemma 4.2 of [Mo]. It is presented next for the reader's convenience. Let $f: T \cup D \rightarrow[0,1]$ be a continuous function such that $f^{-1}(\{0\})=Z$. Define $W_{m, n}:=\left\{\mathbf{x} \in Z: V(\mathbf{x}, m) \subset f^{-1}([0,1 / n))\right\}$. Note that $\bigcup_{m} W_{m, n}=Z$ for all positive $n \in \mathbb{N}$. Furthermore, $Z=$ $E \backslash \bigcup_{n}\{f>1 / n\}$. Let $C_{m, n}$ be the euclidean closure of $W_{m, n}$ in $E$. Then $C_{m, n} \cap\{f>1 / n\}=\emptyset$. For suppose that there exists a positive $n \in \mathbb{N}$ for which this intersection is nonempty. Pick $\mathbf{y} \in C_{m, n} \cap\{f>1 / n\}$. Choose $p \in \mathbb{N}$ with $V(\mathbf{y}, p) \subset\{f>1 / n\}$. Since both $p$ and $m$ are fixed natural numbers, we can select $\mathbf{x}_{k} \in W_{m, n}$ close enough to $\mathbf{y}$ (in the euclidean topology) so that $V(\mathbf{y}, p) \cap V\left(\mathbf{x}_{k}, m\right) \neq \emptyset$. But this contradicts the fact that $V\left(\mathbf{x}_{k}, m\right) \subset W_{m, n} \subset\{f<1 / n\}$ and $V(\mathbf{y}, p) \subset\{f>1 / n\}$. Thus $\left(\bigcap_{n} \bigcup_{m} C_{m, n}\right) \cap\left(\bigcup_{n}\{f>1 / n\}\right)=\emptyset$. Since

$$
Z=\left(\bigcap_{n} \bigcup_{m} W_{m, n}\right) \subset\left(\bigcap_{n} \bigcup_{m} C_{m, n}\right) \subset E \backslash \bigcup_{n}\{f>1 / n\}=Z,
$$

and $\bigcap_{n} \bigcup_{m} C_{m, n}$ is a euclidean Borel subset of $E$, so is $Z$. Thus, the same happens with all the Baire subsets of $E$.

Finally, we show that $T \cup D$ is not quasi-Mař́k. For every $B \in \mathrm{Ba}(T \cup D)$, let $\nu(B):=\lambda^{*}(E \cap B)$, where $\lambda$ is the linear Lebesgue measure on $D$. By the previous argument $E \cap B$ is a euclidean Borel subset of $E$, so $\nu$ is a well-defined Baire measure on $T \cup D$. Now the subspace topology of $D$ is discrete, and $\nu(E)>0$. By Theorem 14.7 (ii) of [Ku], the cardinality of $E$ is not real-valued measurable. Therefore, $\nu$ has no extension to $\operatorname{Bo}(T \cup D)$.

3.9. Remark. While Kunen's theorem (asserting that Lebesgue measure cannot be extended in a nontrivial way to all subsets of $E$ if $E$ is nonmeasurable of least possible cardinality) remains unpublished, there are stronger results in the literature. For instance E. Grzegorek shows in [Grz], Corollary 2, that there exist two subsets $A$ and $B$ of the real line, one of universal measure zero and the other nonmeasurable, with the same cardinality. It is easy to see (and also well known) why this implies Kunen's result: Just note that if Lebesgue measure could be extended in a nontrivial way to all subsets of a nonmeasurable set $D$ with cardinality less than or equal to the cardinality of a set $A$ of universal measure zero, then any injection from $D$ into $A$ would produce a nontrivial continuous Borel measure on $A$, and we would have a contradiction. Thus, a nonmeasurable $D$ of least possible cardinality cannot be real-valued measurable.

Next we consider the behavior under quasiperfect maps of the Mařík property. A continuous onto map $f: X \rightarrow Y$ is perfect if it is closed and all its fibers are compact (i.e., $f^{-1}(y)$ is compact for every $y \in Y$ ), and quasiperfect if it is closed and all its fibers are countably compact. Every space $X$ 
is the image under a perfect irreducible map of an extremally disconnected space $E(X)$, called the absolute of $X$. In [Wh2], R. F. Wheeler asked whether it is true that $X$ is Mař́k iff $E(X)$ is Mař́k. H. Ohta and K. Tamano gave negative answers to both implications (Examples 3.6 and 3.7 of [OT]; the second example uses the special assumption that the cardinal $c$ of the continuum is not real-valued measurable). So the Mařík property is not preserved by perfect maps in either direction. However, if the perfect map is also open, then the image of a Mařík space is Mařík (Corollary 4.16 of [OT]). H. Ohta and K. Tamano asked whether the preimage under an open perfect map of a Mařík space must also be Mařík. Next we show that this is the case.

We shall use the following theorem (whose proof can be found in [BB], Theorem 3.6.1): Let $\mathcal{A}_{1}$ and $\mathcal{A}_{2}$ be two algebras of sets on $X$, and let $\mu_{1}$ and $\mu_{2}$ be finite, finitely additive measures defined on $\mathcal{A}_{1}$ and $\mathcal{A}_{2}$ respectively. Let $\mathcal{B}$ be any algebra containing both $\mathcal{A}_{1}$ and $\mathcal{A}_{2}$. A necessary and sufficient condition for the existence on $\mathcal{B}$ of a finite, finitely additive measure which is a common extension of $\mu_{1}$ and $\mu_{2}$ is the following: Whenever $A_{1} \in \mathcal{A}_{1}$ and $A_{2} \in \mathcal{A}_{2}$, if $A_{1} \subset A_{2}$, we have $\mu_{1}\left(A_{1}\right) \leq \mu_{2}\left(A_{2}\right)$, while if $A_{2} \subset A_{1}$, then $\mu_{1}\left(A_{1}\right) \geq \mu_{2}\left(A_{2}\right)$.

The next result is a special case of Lemma 3.4 of [Fro].

3.10. Lemma. Let $f$ be an open quasiperfect map from $X$ onto $Y$. If $Z$ is a zero subset of $X$, then $f(Z)$ is a zero subset of $Y$.

The following lemma is well known and easy to prove.

3.11. Lemma. Let $f$ be a quasiperfect map from $X$ onto $Y$. If $\left\{F_{n}\right\}$ is a sequence of closed subsets of $X$ such that $F_{n} \downarrow \emptyset$, then $\left\{f\left(F_{n}\right)\right\}$ is a sequence of closed subsets of $X$ with $f\left(F_{n}\right) \downarrow \emptyset$.

One direction of the following theorem is Corollary 4.16 of [OT] (though there it is stated for perfect rather than quasiperfect maps) so we only need to prove the other implication.

3.12. TheOREM. Let $f$ be an open quasiperfect map from $X$ onto $Y$. Then $X$ is Mařik iff $Y$ is Mařik.

Proof. Let $f$ be an open and quasiperfect map from $X$ onto the Mařík space $Y$, and let $\nu$ be a Baire measure on $X$. Let $\mu$ be any regular Borel extension of the Baire measure $\nu\left(f^{-1}(\cdot)\right)$ on $Y$. Denote by $\mathcal{A}(\mathcal{F}(X))$ the algebra generated by the closed sets of $X$, i.e., the Borel algebra of $X$. Then $\mathrm{Ba}(X) \cap \mathcal{A}(\mathcal{F}(X))$ and $\left\{f^{-1}(B): B \in \operatorname{Bo}(X)\right\} \cap \mathcal{A}(\mathcal{F}(X))$ are subalgebras of $\mathcal{A}(\mathcal{F}(X))$, $\nu$ is (by restriction) a measure on $\operatorname{Ba}(X) \cap \mathcal{A}(\mathcal{F}(X))$, and $\mu_{f}(\cdot):=\mu(f(\cdot))$ is a measure on $\left\{f^{-1}(B): B \in \operatorname{Bo}(X)\right\} \cap \mathcal{A}(\mathcal{F}(X))$. Let $A_{1} \in \mathrm{Ba}(X) \cap \mathcal{A}(\mathcal{F}(X))$ and $A_{2} \in\left\{f^{-1}(B): B \in \operatorname{Bo}(X)\right\} \cap \mathcal{A}(\mathcal{F}(X))$. Suppose $A_{1} \subset A_{2}$. By Lemma 3.10, if $Z$ is a zero subset of $X$, then $f(Z)$ is a zero subset of $Y$. Now $A_{2}=f^{-1}(B)$ for some $B \in \operatorname{Bo}(X)$, so if $Z \subset A_{1}$, then 
$f(Z) \subset B$ and $f^{-1}(f(Z)) \subset f^{-1}(B)$. Taking suprema over all $Z \in \mathcal{Z}(X)$ with $Z \subset A_{1}$, we obtain

$$
\nu\left(A_{1}\right)=\sup \nu(Z) \leq \sup \nu\left(f^{-1}(f(Z))\right)=\sup \mu_{f}\left(f^{-1}(f(Z))\right) \leq \mu_{f}\left(A_{2}\right) .
$$

Suppose next that $A_{2}=f^{-1}(B) \subset A_{1}$. Let $O$ be a cozero subset of $X$ with $A_{1} \subset O$. Then $f\left(O^{\mathrm{c}}\right)$ is a zero subset of $Y$, disjoint from $B$. Hence $\nu(X)=$ $\mu(Y) \geq \mu(B)+\mu\left(f\left(O^{\mathrm{c}}\right)\right)=\mu_{f}\left(f^{-1}(B)\right)+\nu\left(f^{-1}\left(f\left(O^{\mathrm{c}}\right)\right)\right) \geq \mu_{f}\left(A_{2}\right)+\nu\left(O^{\mathrm{c}}\right)$. Therefore $\mu_{f}\left(A_{2}\right) \leq \nu(X)-\nu\left(O^{\mathrm{c}}\right)=\nu(O)$. By the regularity of $\nu$ it follows that $\mu_{f}\left(A_{2}\right) \leq \nu\left(A_{1}\right)$. The result mentioned above (Theorem 3.6.1 of [BB]) entails that there is a common extension $\psi$ of $\nu$ and $\mu_{f}$ to $\mathcal{A}(\mathcal{F}(X))$. The rest of the argument follows the same lines as the proof of Theorem 2.1: There exists a finitely additive regular measure $\phi$ on $\mathcal{A}(\mathcal{F}(X))$ with $\phi(X)=\psi(X)$ and $\phi \geq \psi$ on the closed subsets of $X$. As in Theorem 2.1, using the regularity of $\nu$ we conclude that $\phi$ extends $\nu$ (to be precise, it extends $\nu$ restricted to $\mathrm{Ba}(X) \cap \mathcal{A}(\mathcal{F}(X)))$, and an identical argument, using closed sets instead of zero sets, shows that $\phi$ is also an extension of $\mu_{f}$ on $\left\{f^{-1}(B): B \in \operatorname{Bo}(X)\right\} \cap \mathcal{A}(\mathcal{F}(X))$. But now, for every sequence $\left\{F_{n}\right\}$ of closed sets with $F_{n} \subset X$ and $F_{n} \downarrow \emptyset$, we have $\left\{f\left(F_{n}\right)\right\} \downarrow \emptyset$ (Lemma 3.11), so from the countable additivity of $\mu$ it follows that $\lim _{n} \phi\left(F_{n}\right) \leq \lim _{n} \phi\left(f^{-1}\left(f\left(F_{n}\right)\right)\right)=\lim _{n} \mu\left(f\left(F_{n}\right)\right)=0$. The regularity of $\phi$ entails the same conclusion for arbitrary decreasing sequences of sets in $\mathcal{A}(\mathcal{F}(X)$ ) with empty intersection, so there exists a regular Borel (countably additive) measure extending $\phi$.

H. Ohta and K. Tamano have proven (Theorem 4.17 of $[\mathrm{OT}]$ ) that the preimage $X$ under a quasiperfect map of a Baire separated, Mař́k space $Y$ is Mařík, and hence that the product of a compact space with a Baire separated, Mařík space is Mař́k (Corollary 4.19 of [OT]). The result given above entails that the Baire separation of $Y$ can be replaced by the condition that the map be open, thus answering in the affirmative Questions 4.20 of $[\mathrm{OT}]$.

3.13. Corollary. Let $f$ be an open perfect map from $X$ onto $Y$. If $Y$ is Mařik, then so is X. In particular, the product of a Mařik space with a compact space is Mařik.

Proof. Recall that if one of the factors in a product is compact, then the projection onto the other is perfect and open. So the product of a Mařík space with a compact space is Mařík.

If the product is Baire separated, the compactness of one of the factors can be relaxed to strong measure compactness. Given a Baire measure $\nu$ on $A \subset X$, its natural extension $\nu_{X}$ to $\mathrm{Ba}(X)$ is defined by $\nu_{X}(B):=\nu(B \cap A)$ for every $B \in \mathrm{Ba}(X)$. If $A$ is Baire embedded in $X$ and $\mu_{X}$ is a Baire measure on $X$, its natural restriction $\mu$ to $\mathrm{Ba}(A)$ is defined as follows: $\mu(B)=\mu_{X}^{*}(B)$ 
for all $B \in \mathrm{Ba}(A)$, where $\mu_{X}^{*}$ is the outer measure generated by $\mu$. Note that if $\mu_{X}(X)=\mu_{X}^{*}(A)$, then $\mu(B)=\mu_{X}\left(B_{1}\right)$, where $B_{1}$ is any Baire subset of $X$ which satisfies $B=B_{1} \cap A$. The measure $\mu$ is well defined, since if $B_{1}, B_{2} \in \operatorname{Ba}(X)$ with $B=B_{1} \cap A=B_{2} \cap A$, then $B_{1} \triangle B_{2} \subset A^{\mathrm{c}}$, so $\mu_{X}\left(B_{1} \triangle B_{2}\right)=0$. The definition of natural extensions and restrictions for regular Borel measures is entirely analogous.

3.14. Proposition. Let $X \times Y$ be a Baire separated space, where $X$ is Mařik and $Y$ is strongly measure compact. Then $X \times Y$ is Mařik.

Proof. By Lemma 4.5 of [OT], the Baire separation of $X \times Y$ entails that its $F_{\sigma}$ subsets are Baire embedded and generalized Baire in $X \times Y$. Let $\nu$ be a Baire measure on $X \times Y$. For each $n \in \mathbb{N}$, select a compact set $K_{n} \subset Y$ such that $K_{n-1} \subset K_{n}$ and $\nu^{*}\left(\pi_{Y}^{-1}\left(K_{n}\right)\right)>\nu(X \times Y)-1 / n$, where $\pi_{Y}$ is the projection from $X \times Y$ onto $Y$. Note that $\bigcup_{n} X \times K_{n}$ is $F_{\sigma}$ in $X \times Y$, so it is Baire embedded and generalized Baire in $X \times Y$. Clearly, each $X \times K_{n}$ is also Baire embedded and generalized Baire in $\bigcup_{n} X \times K_{n}$. Furthermore, each set $X \times K_{n}$ is Mař́k (Corollary 3.13), whence $\bigcup_{n} X \times K_{n}$ is Mař́k by Theorem 4.9 of $[\mathrm{OT}]$. Let $B \in \mathrm{Ba}\left(\bigcup_{n} X \times K_{n}\right)$. For each $n$ select $B_{n} \in \mathrm{Ba}(X \times Y)$ with $B_{n} \cap\left(X \times K_{n}\right)=B \cap\left(X \times K_{n}\right)$. It is easy to check, using the fact that $\left\{X \times K_{n}\right\}$ is an increasing sequence of sets, that $\left(\bigcap_{n=1}^{\infty} \bigcup_{k=n}^{\infty} B_{k}\right) \cap\left(\bigcup_{n} X \times K_{n}\right)=B$. Since $\bigcap_{n=1}^{\infty} \bigcup_{k=n}^{\infty} B_{k} \in \operatorname{Ba}(X \times Y)$, it follows that $\bigcup_{n} X \times K_{n}$ is Baire embedded in $X \times Y$. Denote by $\phi$ the natural restriction of $\nu$ to $\bigcup_{n} X \times K_{n}$, and let $\mu$ be a regular Borel extension of $\phi$. Then the natural extension $\mu_{X \times Y}$ of $\mu$ to $X \times Y$ is an extension of $\nu$, and since $\bigcup_{n} X \times K_{n}$ is $F_{\sigma}$ in $X \times Y$, it follows that $\mu_{X \times Y}$ is regular.

If a continuous onto map is open and has compact fibers, but is not closed, then the preimage of a Mařík space may fail to be Mařík. To see this, consider Nemytski1's tangent disc topology (see Example 82 of [SS]) on the unit square $[0,1]^{2}$. The projection onto the first coordinate is continuous, open, and has compact fibers. Since the subspace $[0,1] \times\{0\}$ has the discrete topology, trivially it is Mařík. For the same reasons as in Lemma 4.2 of [Mo] (or in the proof of our Theorem 3.8), linear Lebesgue measure on $[0,1] \times\{0\}$ defines a Baire measure $\lambda$ on the Nemytskiu square $[0,1]^{2}$. Assuming that $c$ is not real-valued measurable, $\lambda$ has no Borel extension. A small modification of this example allows us to do away with the previous set-theoretic assumption: Rather than $[0,1]^{2}$, use $E \times[0,1]$, where $E \subset[0,1]$ is nonmeasurable (with respect to Lebesgue measure) of least possible cardinality. Then apply the result of Kunen mentioned above (Theorem 14.7(ii) of [Ku]).

There remain several open questions from $[\mathrm{OT}]$ which are unanswered here. In this author's opinion the most interesting ones are whether $\mathbb{N}^{\lambda}$ is Mař́k for every cardinal $\lambda$ (it is quasi-Mařík by Cor. 2.12 of [OT]) and 
whether the realcompactification of a Mařík space must be Mařík (Questions 2.13 and 2.14, p. 401 of [OT]). Under Martin's Axiom plus the negation of the Continuum Hypothesis, D. H. Fremlin has proven (in [F3]) that for every $\lambda<c, \mathbb{N}^{\lambda}$ is measure compact and hence Mařík. But E. K. van Douwen showed ([vD], Theorem 13.7, p. 109) that $\mathbb{N}^{\lambda}$ is not countably metacompact when $\lambda$ is uncountable ( $\mathrm{I}$ am indebted to Professor $\mathrm{H}$. Ohta for pointing out this reference). This leaves $\mathbb{N}^{\lambda}$ with $\lambda \geq c$ as a possible example in ZFC of a quasi-Mařík space which is not Mařík. As noted in [OT], showing that $\mathbb{N}^{\lambda}$ is not Mařík for some $\lambda \geq c$ would also provide an example of a Mařík space without a Mařík realcompactification. Van Douwen's proof showing that countable metacompactness fails for $\mathbb{N}^{\omega_{1}}$ is rather indirect, so we give below a direct proof due to M. E. Rudin. It is presented here with her kind permission.

Let us fix some notation. By $\lambda$ we denote a cardinal. If $g$ is a partial function on $\lambda$ (i.e., defined on a subset of $\lambda$ ), with values in $\mathbb{N}$, then $[g]$ denotes the set $\left\{f \in \mathbb{N}^{\lambda}: f(\alpha)=g(\alpha)\right.$ for every $\left.\alpha \in \operatorname{Dom} g\right\}$. Given a set $A$, we write card $A$ for its cardinality. The family of all sets $[g]$, where $g$ is a finite partial function, forms a base for the topology of $\mathbb{N}^{\lambda}$. We follow the convention of identifying an ordinal with the set of all its predecessors.

\subsection{TheOREM. The space $\mathbb{N}^{\omega_{1}}$ is almost Dowker.}

Proof. Since $\mathbb{N}^{\omega_{1}}$ is completely regular, it is enough to show that it is not countably metacompact. Set $F_{n}:=\left\{f \in \mathbb{N}^{\omega_{1}}: \operatorname{card}\{f(\alpha)=i\} \leq 1\right.$ for $i \leq n\}$. Then $\left\{F_{n}\right\}$ is a decreasing sequence of closed sets with empty intersection. Let $\left\{O_{n}\right\}$ be a sequence of open sets with $F_{n} \subset O_{n}$. We shall see that $\bigcap_{n} O_{n} \neq \emptyset$. Denote by $G$ the set of all finite partial functions from $\omega_{1}$ into $\mathbb{N}$. Pick any injective function $h_{0} \in G$. Select, for every $\alpha<\omega_{1}$, an $f_{\alpha}^{1} \in F_{1}$ such that $f_{\alpha}^{1}$ is an extension of $h_{0}$, and the restriction of $f_{\alpha}^{1}$ to $\alpha$ is injective. Choose next $g_{\alpha}^{1} \in G$ with $h_{0} \subset g_{\alpha}^{1}$ and $f_{\alpha}^{1} \in\left[g_{\alpha}^{1}\right] \subset O_{1}$. By the $\Delta$-system lemma there exists an uncountable subset $\Lambda$ of $\omega_{1}$ such that $\left\{\right.$ Dom $\left.g_{\alpha}^{1}: \alpha \in \Lambda\right\}$ forms a $\Delta$-system, i.e., there exists a fixed set $r$ (the root) such that given two different functions $g_{\alpha}^{1}$ and $g_{\beta}^{1}$ with $\alpha, \beta \in \Lambda$, we have Dom $g_{\alpha}^{1} \cap \operatorname{Dom} g_{\beta}^{1}=r$. Furthermore, there is an uncountable $\Lambda_{1} \subset \Lambda$ such that for all $\alpha, \beta \in \Lambda_{1}$, we have $g_{\alpha}^{1}=g_{\beta}^{1}$ on $r$ (otherwise, $\Lambda$ would be countable). Let $H_{1}$ be the set $\left\{g_{\alpha}^{1}: \alpha \in \Lambda_{1}\right\}$. Then $H_{1}$ is a $\Delta$-system, with root, say, $h_{1}$. Note that $h_{0} \subset h_{1}$. Also, $h_{1}$ is injective, since for any $\alpha \in \Lambda_{1}$ with $\alpha>\max \operatorname{Dom} h_{1}$, the function $f_{\alpha}^{1}$ extends $h_{1}$ and is injective below $\alpha$. Inductively we produce for each $n \in \mathbb{N}$ a $\Delta$-system $H_{n}$ with an injective root $h_{n}$ which extends $h_{n-1}$, and such that if $w \in H_{n}$, then $[w] \subset O_{n}$. Let $\gamma_{0}=\sup \left\{\bigcup_{n} \operatorname{Dom} h_{n}\right\}$. Since $\gamma_{0}$ is countable, and every $\alpha \in \gamma_{0} \backslash \operatorname{Dom} h_{1}$ belongs to the domain of at most one element of $H_{1}$, it follows that there exists a $g_{1} \in H_{1}$ such that if $\alpha \in \operatorname{Dom} g_{1} \backslash \operatorname{Dom} h_{1}$, then $\alpha>\gamma_{0}$. Let 
$\gamma_{1}=\sup \left\{\left(\bigcup_{n} \operatorname{Dom} h_{n}\right) \cup\left(\operatorname{Dom} g_{1}\right)\right\}$ and choose $g_{2} \in H_{2}$ with $\alpha>\gamma_{1}$ for all $\alpha \in \operatorname{Dom} g_{2} \backslash$ Dom $h_{2}$. Repeating this process, we obtain a sequence $\left\{g_{n}\right\}$ such that $\bigcup_{n} g_{n}$ is a well-defined partial function. Since $\left[g_{n}\right] \subset O_{n}$, it follows that $\emptyset \neq\left[\bigcup_{n} g_{n}\right] \subset \bigcap_{n} O_{n}$.

In [St], A. H. Stone proved that a cartesian product of metric spaces is normal iff it is paracompact iff all but countably many of the factors are compact. K. Nagami [Na] showed that "paracompact" could be replaced by "countably paracompact". By van Douwen's result, we have

3.16. Corollary. A cartesian product of metric spaces is countably metacompact iff all but countably many of the factors are compact.

Proof. One direction is immediate from Stone's Theorem, since paracompactness is a stronger condition than countable metacompactness. For the other, note that if uncountably many factors are not compact, then $\mathbb{N}^{\omega_{1}}$ embeds as a closed subset in the product. Since a closed subset of a countably metacompact set is countably metacompact, the result follows.

Thus we see that for products of metric spaces, the notions of paracompactness, metacompactness, countable paracompactness and countable metacompactness are all equivalent, since the stronger and the weaker conditions coincide.

Note also that for any uncountable product of topological (not necessarily metric) spaces, if $\mathbb{N}$ embeds as a closed discrete subset in uncountably many of the factors, then the product is not countably metacompact. The reason, as before, is that in this case $\mathbb{N}^{\omega_{1}}$ embeds as a closed subset of the product.

3.17. Remark. It is still unknown whether realcompact Dowker spaces exist in ZFC (Question 4, p. 185 of [Ru2]). We see, however, that realcompact almost Dowker spaces abound. Let $\lambda$ be uncountable. Given $\mathbb{R}^{\lambda}$, we see that $\Sigma:=\left\{f \in \mathbb{R}^{\lambda}: \operatorname{card}\{f(\alpha) \neq 0\} \leq \omega\right\}$ is a $\Sigma$-product of $\mathbb{R}^{\lambda}$. As H. Ohta and K. Tamano point out in the proof of Corollary 2.12 of [OT], this $\Sigma$ is a normal, countably paracompact space whose realcompactification is $\mathbb{R}^{\lambda}$. Hence, the realcompactification of a normal, countably paracompact space can be almost Dowker.

\section{References}

[Ad1] W. Adamski, On regular extensions of contents and measures, J. Math. Anal. Appl. 127 (1987), 211-225.

[Ad2] - On the interplay between a topology and its associated Baire and Borel $\sigma$ algebra, Period. Math. Hungar. 21 (2) (1987), 85-93.

[Ad3] —, $\tau$-smooth Borel measures on topological spaces, Math. Nachr. 78 (1977), 97107. 
[Ba] W. Bade, Two properties of the Sorgenfrey plane, Pacific J. Math. 51 (1974), 349-354.

[Be] M. G. Bell, On the combinatorial principle P(c), Fund. Math. 114 (1981), 149157.

[BB] K. P. S. Bhaskara Rao and M. Bhaskara Rao, Theory of Charges, Academic Press, 1983.

[vD] E. K. van Douwen, Covering and separation properties of box products, in: Surveys in General Topology, G. M. Reed (ed.), Academic Press, 1980, 55-129.

[Do] C. H. Dowker, On countably paracompact spaces, Canad. J. Math. 3 (1951), 219-224.

[Eng] R. Engelking, General Topology, Heldermann, Berlin, 1989.

[F1] D. H. Fremlin, Consequences of Martin's Axiom, Cambridge Univ. Press, 1984.

[F2] -, Real-valued-measurable cardinals, in: Set Theory of the Reals, H. Judah (ed.), Israel Math. Conf. Proc. 6, Bar-Ilan Univ., Ramat Gan, 1993, 151-304.

[F3] -, Uncountable powers of $\mathbb{R}$ can be almost Lindelöf, Manuscripta Math. 22 (1977), 77-85.

[Fro] Z. Frolík, Applications of complete families of continuous functions to the theory of Q-spaces, Czechoslovak Math. J. 11 (1961), 115-133.

[GJ] L. Gillman and M. Jerison, Rings of Continuous Functions, Van Nostrand, Princeton, N.J., 1989.

[Grz] E. Grzegorek, Solution of a problem of Banach on $\sigma$-fields without continuous measures, Bull. Acad. Polon. Sci. Sér. Sci. Math. 28 (1980), 7-10.

[HRR] A. W. Hager, G. D. Reynolds and M. D. Rice, Borel-complete topological spaces, Fund. Math. 75 (1972), 135-143.

[Ish] F. Ishikawa, On countably paracompact spaces, Proc. Japan Acad. 31 (1955), 686-687.

[Ka] A. Kato, Union of realcompact spaces and Lindelöf spaces, Canad. J. Math. 31 (1979), 1247-1268.

[Ki] R. B. Kir k, Locally compact, B-compact spaces, Indag. Math. 31 (1969), 333-344.

[Kn] J. D. Knowles, Measures on topological spaces, Proc. London Math. Soc. (3) 17 (1967), 139-156.

$[\mathrm{Ku}] \mathrm{K} . \mathrm{Kunen}$, Inaccessibility properties of cardinals, Ph.D. thesis, Stanford Univ., 1968.

[Lem] J. Lembcke, Konservative Abbildungen und Fortsetzung regulärer Masse, Z. Wahrsch. Verw. Gebiete 15 (1970), 57-96.

[Ma] J. Mařík, The Baire and Borel measure, Czechoslovak Math. J. 7 (1957), 248253.

[Mo] W. Moran, The additivity of measures on completely regular spaces, J. London Math. Soc. 43 (1968), 633-639.

[Na] K. Nagami, Countable paracompactness of inverse limits and products, Fund. Math. 73 (1972), 261-270.

[OT] H. Ohta and K. Tamano, Topological spaces whose Baire measure admits a regular Borel extension, Trans. Amer. Math. Soc. 317 (1990), 393-415.

[Ost] A. J. Ostaszewski, On countably compact, perfectly normal spaces, J. London Math. Soc. (2) 14 (1976), 505-516.

[Ox] J. C. Oxtoby, Homeomorphic measures in metric spaces, Proc. Amer. Math. Soc. 24 (1970), 419-423.

[Pa] J. K. Pachl, Disintegration and compact measures, Math. Scand. 43 (1978), $157-168$. 
[Ru1] M. E. Rudin, Dowker spaces, in: Handbook of Set-Theoretic Topology, K. Kunen and J. E. Vaughan (eds.), North-Holland, 1984, 761-780.

[Ru2] -, A normal space $X$ for which $X \times I$ is not normal, Fund. Math. 73 (1971), 179-186.

[S] B. M. Scott, Some "almost-Dowker" spaces, Proc. Amer. Math. Soc. 68 (1978), 359-364.

[SS] L. A. Steen and J. A. Seebach, Counterexamples in Topology, Springer, 1986.

[St] A. H. Stone, Paracompactness and product spaces, Bull. Amer. Math. Soc. 58 (1948), 977-982.

[Sz] P. J. Szeptycki, Dowker spaces, in: Topology Atlas 1, D. Shakhmatov and S. Watson (eds.), electronic publication, 1996, 45-47.

[T] F. Topsøe, On construction of measures, in: Proc. Conf. "Topology and Measure I" (Zinnowitz 1974), Part 2, J. Flachsmeyer, Z. Frolík and F. Terpe (eds.), Ernst-Moritz-Arndt Univ., Greifswald, 1978, 343-381.

[U] S. Ulam, Zur Masstheorie in der allgemeinen Mengenlehre, Fund. Math. 16 (1930), 140-150.

[W] W. Weiss, Versions of Martin's axiom, in: Handbook of Set-Theoretic Topology, K. Kunen and J. E. Vaughan (eds.), North-Holland, 1984, 827-886.

[Wh1] R. F. Wheeler, A survey of Baire measures and strict topologies, Exposition. Math. 77 (1983), 97-190.

[Wh2] - Extensions of a $\sigma$-additive measure to the projective cover, in: Lecture Notes in Math. 794, Springer, 1980, 81-104.

Departamento de Matemáticas

Facultad de Ciencias

Universidad Autónoma de Madrid

28049 Madrid, Spain

E-mail: aldaz@ccuam3.sdi.uam.es 